Baltic Astronomy, vol. 6, 192-195, 1997.

\title{
THE CENTRE DE DONNÉES ASTRONOMIQUES DE STRASBOURG
}

\author{
F. Genova, F. Bonnarel, J. G. Bartlett, P. Dubois, D. Egret,
} G. Jasniewicz, S. Lesteven, F. Ochsenbein and M. Wenger

CDS, URA CNRS 1280, Observatoire de Strasbourg, 11 me de l'Université, Strasbourg 67000, France

Received July 18, 1996.

\begin{abstract}
The activities of the Centre de Données astronomiques de Strasbourg (CDS) are reviewed. They include: SIMBAD, the reference database for the identification and bibliography of astronomical objects, the catalog service with the new VizieR Catalogue Browser developed as a joint effort by CDS and ESA/ESRIN and the WWW server. The future evolution of these services, including the distribution of the ALADIN interactive sky atlas, is also assessed.
\end{abstract}

Key words: information retrieval services - databases

The objectives of the Centre de Données astronomiques de Strasbourg (CDS) can be summarized as follows: to collect, homogenize, distribute and preserve astronomical information for the use of the entire astronomical community. In practical terms, this means that the CDS defines, develops and distributes tools to help astronomers to retrieve the information they need, among the vast and rapidly growing array of possible sources.

At the present time, the CDS maintains several services:

(1) SIMBAD, the reference database for the identification and bibliography of the astronomical objects (controlled access requiring a userid and password). SIMBAD presently has about 2700 users in 50 countries. 
SIMBAD contains the identifications, "basic data", bibliography and some measurements for astronomical objects, taken from the papers published in journals and from selected catalogs. In May 1996, SIMBAD contained 1225000 objects (up by $65 \%$ compared with 1990), with more than 1700 different possible names and 3773000 object names. The Paris, Bordeaux and Strasbourg Observatories participate in the bibliography scanning, under the responsibility of the Institut d'Astrophysique de Paris. 90 journals are systematically scanned, and the database contained 92000 references in May 1996 (up by $60 \%$ compared with 1990), with 1795000 object citations. In parallel, large catalogs are systematically included in the database, to improve the multi-wavelength coverage, and/or linked to incoming large projects, in collaboration with external specialized teams (e.g. ARI, Heidelberg; Paris Observatory - DASGAL; Besançon Observatory; INASAN). In recent years, a large effort was devoted to the inclusion of stellar catalogs (PPM, HIC, CCDM) for the HIPPARCOS mission, of the IRAS PSC and IUE log and to the "cleaning" of the Einstein and older X-ray catalogs.

(2) The Catalogue Server and the new Catalogue Browser VizieR.

The Catalogue Server displays catalogs and published tables. The collection of catalogs and tables is a cooperation between the Data Centers: CDS, ADC, INASAN, NAOJ and Beijing Observatory. A general standard is shared by the Data Centers and publishers (Astron. \& Astrophys., the AAS CD-ROM tables; and now Astronomicheskij Zhurnal) (Ochsenbein 1997). In June 1996, the CDS service contained 1550 catalogs and tables (650 in 1993), among which 1050 are accessible on line with a standard description. The catalogs and tables can be queried by keywords from the WWW or via anonymous FTP on cdsarc.u-strasbg.fr or 130.79.128.5 (directory pub/cats). This allows one to retrieve whole catalogs. The new VizieR Catalogue Browser, developed in 1995 in collaboration with ESA/ESRIN, as a follow-up of the ESIS Catalogue Browser, has been accessible from the WWW since February 1996. It allows one to query any table or catalog by any of its fields.

(3) The CDS WWW server (URL http://cdsweb.u-strasbg . $\mathrm{fr} / \mathrm{CDS} . \mathrm{html}$ ) gives access to the "Dictionary of nomenclature of astronomical objects" (Lortet et al. 1994), to bibliography information and to documentation. It also gives access to some services hosted by the CDS, "Yellow Page" services such as the Star*s Family, which 
was developed by Heck (1995), and AstroWeb (Jackson et al. 1994), which is the TOPBase atomic physics database from the OPACITY project (Cunto et al. 1993).

The CDS also hosts ULDA (as the French national host), the archives of unpublished data on variable stars (IAU Commission 27) and other information. The Bulletin d'Information du CDS, published twice a year, contains in particular refereed papers describing catalogs deposited at CDS, which were not published in other refereed journals. A hotline at email question@simbad.u-strasbg.fr is available, for any question on, or comments about, the CDS services. It is particularly important that users can point out the errors which they find in the services.

The ALADIN interactive sky atlas provides deep images from the digitized sky, with astrometric and photometric calibration, on which information from SIMBAD, from the CDS catalogs and tables, as well as from tables provided by users, can be overlaid. It will thus be a major tool for multi-wavelength cross-identification. Images with full resolution ( $\left.0.7^{\prime \prime}\right)$ from the MAMA digitizing machine, and soon from SUPERCOSMOS, are provided for "crowded fields", such as the Galactic plane or the Magellanic Clouds. The whole sky is covered by the compressed STScI Sky Survey CD-ROMs (1.8" resolution). ALADIN is reaching the end of its development phase, and will be distributed to the French astronomy laboratories by the end of 1996 , as a first step.

Medium term evolution includes:

- The building of a transparent access to the CDS services on the WWW, including a WWW version of SIMBAD. A general CDS data exchange model has been built, which permits navigation between the services.

- The building of access to distributed data archives from VizieR, by including observatory logs in the system. Standard anchors and requests to archives are presently being defined (with ESO, CADC, IUE and OAT).

- Increased links with astronomical publications. CDS already builds electronic tables for Astron. \& Astrophys. and installs the tables from the AAS CD-ROMs on-line, by agreement with the journal editors. It will soon host a clone of the ADS bibliographic database. In the future, innovative new services will certainly develop, owing to an increased synergy between databases and electronic journals. 
- Continuation of the development of mirror catalog archives between the Data Centers.

\section{REFERENCES}

Cunto W., Mendoza C., Ochsenbein F., Zeippen C. J. 1993, A\&A, 275, L5 Heck A. 1995, in Information and On-line Data in Astronomy, eds.

D. Egret \& M. A. Albrecht, Kluwer Academic Publishers, p. 195

Jackson R., Wells D., Adorf H.M., Egret D., Heck A., Koekemer A., Murtagh F. 1994, A\&AS, 108, 235

Lortet M.-C., Borde S., Ochsenbein F. 1994, A\&AS, 107, 193

Ochsenbein F. 1997, Baltic Astronomy, 6, 221 